\title{
ESTUDO BIBLIOMÉTRICO SOBRE ILHAS DE CALOR URBANAS E ZONAS CLIMÁTICAS LOCAIS
}

\author{
SOUZA, Camila Amaro de - arq.camila.amaro@gmail.com \\ Universidade Anhanguera/ UNIDERP
}

\author{
PARANHOS FILHO, Antonio Conceição - toniparanhos@gmail.com \\ Universidade Federal de Mato Grosso do Sul/ UFMS \\ GUARALDO, Eliane - arq.artes@gmail.com \\ Universidade Federal de Mato Grosso do Sul/ UFMS
}

\begin{abstract}
RESUMO: A mudança das superfícies naturais pelas áreas urbanas construídas altera os atributos climáticos como a temperatura do ar e a umidade relativa do ar. Desde os anos 1950, as variações térmicas, no que tange os estudos tradicionais de climatologia, são analisadas a partir da diferença entre o campo térmico urbano e o rural, relacionadas à intensidade da ilha de calor urbana (ICU), levando a simplificação desse fenômeno e da realidade paisagística da cidade e seu entorno. O método "Local Climate Zones" (LCZ) de Stewart e Oke (2012) surge como uma tentativa de padronização e avanço no grau de detalhamento das análises de campo térmico a partir da classificação da paisagem. Nesse sentido, os objetivos desse estudo foram: identificar artigos e artigos de revisão publicados utilizando o termo "Urban Heat Island" OR "UHI", inserido em título, resumo e/ ou palavras-chave; identificar a quantidade de artigos e artigos de revisão utilizando o termo "Urban Heat Island* OR "UHI" AND "Local Climate Zones" OR "LCZ", inserido em título, resumo e/ ou palavras-chave e a concentração dessa produção científica por país e por autor; elaborar uma sistematização dos principais critérios adotados nos artigos filtrados para o portfólio bibliográfico. A partir desta pesquisa foi possível interpretar o grau de relevância deste termo para pesquisas em climatologia urbana e obter material de apoio para futuras pesquisas.
\end{abstract}

PALAVRAS-CHAVE: LCZ, classificação da paisagem, campo térmico, bibliometria. ÉTUDE BIBLIOMÉTRIQUE SUR LES ÎLES DE CHALEUR URBAINE ET LES ZONES CLIMATIQUES LOCALES

RESUMMÉ: La modification des surfaces naturelles par les zones urbaines bâties modifie les attributs climatiques tels que la température de l'air et l'humidité relative de l'air. Depuis les années 1950, les variations thermiques dans les études de climatologie traditionnelle sont analysées sour la différence entre les champs thermiques urbains et ruraux, liée à l'intensité de l'îlot de chaleur urbain (ICU), conduisant à la simplification de ce phénomène. et la réalité paysagère de la ville et de ses environs. La méthode des "zones climatiques locales" (LCZ) de Stewart et Oke (2012) apparaît comme une tentative de normalisation et d'amélioration du degré de détail des analyses de champ thermique de la classification de paysage. En ce sens, les objectifs de cette étude étaient les suivants: identifier les articles et les articles de synthèse publiés en utilisant le terme "îlot de chaleur urbain" OU "UHI", insérés dans le titre, le résumé et / ou les mots-clés; identifiez le nombre d'articles et d'articles de synthèse en utilisant les termes "îlot de chaleur urbain * OU" UHI "ET" zones climatiques locales "OU" LCZ ", insérés dans le titre, l'abrégé et / ou les mots-clés, ainsi que la concentration de cette production scientifique par pays et par auteur; élaborer une systématisation des principaux critères retenus dans les articles filtrés pour le portefeuille bibliographique. Depuis cette recherche, il a été possible d'interpréter le degré de pertinence de ce terme pour la recherche en climatologie urbaine et d'obtenir du matériel d'appui pour les recherches futures

MOTS CLÉ: LCZ, classification du paysage, champ thermique, bibliométrie. 
ABSTRACT: The change of natural surfaces by built urban areas changes the climatic attributes such as air temperature and relative humidity. Since the 1950s, thermal variations, with respect to traditional climatology studies, have been analyzed based on the difference between the urban and rural thermal fields, related to the intensity of the urban heat island (ICU), leading to the simplification of this phenomenon, the landscape reality of the city and its surroundings. The "Local Climate Zones" (LCZ) method by Stewart and Oke (2012) appears as an attempt to standardize and advance the degree of detail of thermal field analyzes from the landscape classification. In this sense, the objectives of this study were: to identify articles and review articles published using the term "Urban Heat Island" OR "UHI", inserted in title, abstract and / or keywords; identify the number of articles and review articles using the term "Urban Heat Island * OR" UHI "AND" Local Climate Zones "OR" LCZ ", inserted in title, abstract and / or keywords and the concentration of this scientific production by country and by author; elaborate a systematization of the main criteria adopted in the filtered articles for the bibliographic portfolio. From this research it was possible to interpret the degree of relevance of this term for research in urban climatology and obtain support material for future research.

KEYWORDS: LCZ, landscape classification, thermal field, bibliometry

\section{INTRODUÇÃO}

Climatologia urbana é uma área em vasto crescimento, entretanto, são necessários padrões para garantir intercâmbios consistentes e significativos de dados entre regiões, culturas e áreas temáticas de pesquisa.

Os avanços tecnológicos das últimas décadas têm produzido uma variedade de modelos numéricos para simular os processos-chave que regem os atributos climáticos como: temperatura, umidade relativa do ar e trocas de calor no ambiente urbano (MCCARTHY et al., 2010).

Estudos como os de: MORRIS e SIMMONDS (2000), STEWART (2000), VOOGT e OKE (2003), ARNFIELD (2003), AMORIM (2011), demonstraram que a atmosfera urbana tem apresentado diferenças em relação à atmosfera rural, principalmente no que se refere à temperatura, ocorrendo o desenvolvimento de ilhas de calor.

As ilhas de calor urbanas (ICU), citadas na literatura em inglês como Urban Heat Island (UHI), são definidas tradicionalmente como as variações de temperatura $(\Delta T)$ entre o perímetro urbano e a zona rural, conforme ilustra a Figura 1.

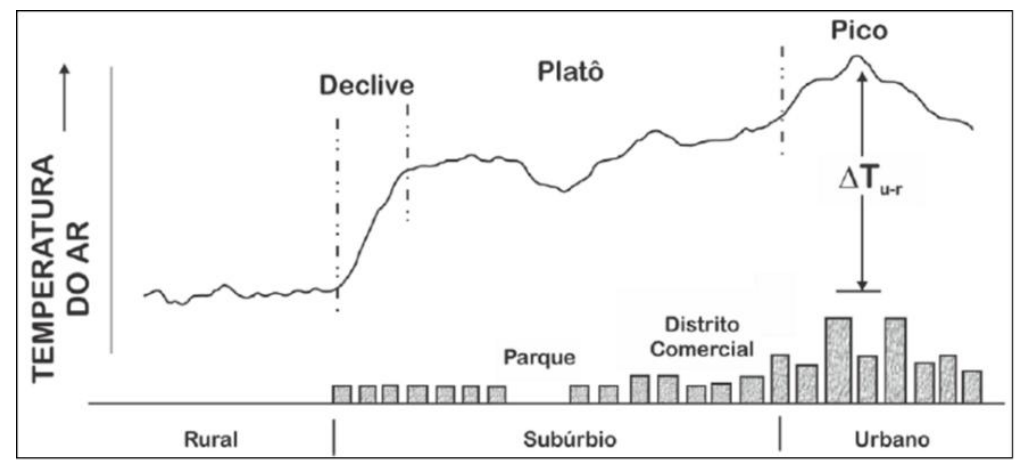

Figura 1- Ilha de calor tradicional, adaptada de Oke (1987).

Isso ocorre devido à morfologia das cidades com abundância de materiais de menor albedo (pavimentação asfáltica, concreto) e carência de cobertura vegetal, acarretando maior emissividade de energia solar para o ambiente 
urbano, o que eleva as temperaturas e cria ilhas de calor causando, consequentemente, desconforto para a população.

As ICUs são geradas por fatores físicos naturais (velocidade dos ventos regionais) e artificiais (utilização de materiais construtivos absorvedores de radiação no período diurno e que liberam o calor no período noturno), sendo estes preponderantes na formação destes fenômenos climáticos.

A característica mais importante a ser observada na ilha de calor urbana é sua intensidade, que geralmente é aferida como a variação máxima entre o ponto mais antropizado da área central da cidade e outro ponto na área rural (AMORIM; DUBREUIL, CARDOSO, 2015). Isso demonstra que a ICU é um fenômeno determinante para as alterações atmosféricas no que se refere a ambientes urbanos.

Podem ser enumeradas diversas causas do efeito ICU, cada causa listada no Quadro 1 representa uma modificação urbana no balanço de energia e radiação da superfície em detrimento da ICU (STEWART e OKE, 2012).

Quadro 1 - Causas do efeito Ilha de Calor Urbana. Cada causa representa uma modificação urbana no balanço de energia e radiação da superfície.

1. Maior absorção de radiação solar devido à reflexão múltipla e captura de radiação pela construção de muros e superfícies verticais na cidade. A maior absorção não é, como frequentemente se supõe, devido exclusivamente ao baixo albedo de materiais urbanos.

2. Maior retenção de radiação infravermelha em desfiladeiros de ruas devido à visão restrita do hemisfério do céu radiativamente "frio". A visão do céu tornase cada vez mais restrita com edifícios mais altos e compactos.

3. Maior absorção e liberação retardada de calor (atraso térmico alto) por edifícios e superfícies pavimentadas na cidade. Muitas vezes incorretamente atribuído apenas às propriedades térmicas dos materiais, este efeito também é devido à "armadilha" de radiação solar e infravermelha e à redução de perdas convectivas na camada de dossel, onde o fluxo de ar é retardado.

4. Maior parte da radiação solar absorvida na superfície é convertida em formas de calor sensível (provocando alteração de temperatura) ao invés de calor latente (que provocaria alteração de estado físico). Este efeito deve-se à substituição de solos úmidos e plantas por superfícies pavimentadas e impermeabilizadas, e como resultado uma diminuição na evaporação da superfície.

5. Maior liberação de calor sensível e latente devido a combustão de combustíveis para transporte urbano, processamento industrial e aquecimento/ resfriamento de espaços domésticos. O calor e a umidade também são liberados pelo metabolismo humano, mas isso geralmente é um componente menor do balanço de energia superficial.

Fonte: Adaptado de Stewart e Oke (2012).

Nos últimos anos, a ciência da climatologia urbana teve um progresso significativo no poder preditivo, as relações gerais entre os critérios principais de alterações térmicas nas cidades estão surgindo e a modelagem cresceu notavelmente, o que fez evoluir muito a comunicação sobre o tema (ARNFIELD, 2003; OKE, 2006).

Contudo, um aspecto ainda não padronizado é a descrição de áreas urbanas e rurais. Para amenizar este problema, foi criado em 2011 o conceito 
de classificação da paisagem Local Climate Zones (LCZ) (STEWART, 2011a; STEWART e OKE, 2012), ou em português: zonas climáticas locais.

As zonas climáticas locais são definidas como "regiões homogêneas em relação a cobertura de solo, estrutura da superfície, material construtivo e atividade humana e que abrangem desde centenas de metros até alguns quilômetros em uma escala horizontal" (STEWART e OKE, 2012, p. 1884). O método permite a elaboração de mapas temáticos em maiores ou menores escalas, e com isso a utilização de imagens de satélite de resolução moderada e gratuita como as LANDSAT (United States Geological Service http://earthexplorer.usgs.gov/) até imagens de alta resolução como as SPOT e Pleiades.

Sua utilização vem sendo realizada principalmente na Europa e na Ásia e, mais recentemente, no nordeste e sudeste do Brasil. Deste modo, existe a demanda de avaliar como se dá a ocorrência dessas publicações e o impacto deste método de classificação para a padronização de estudos na área temática.

Foi realizada análise bibliométrica, um método para analisar quantitativamente publicações acadêmicas aplicando técnicas matemáticas e estatísticas com intuito de medir sua quantidade e desempenho (Pritchard, 1969; Zhang et al., 2015). A bibliometria é comum em análises sistemáticas, abordando várias disciplinas da ciência e engenharias, ao mostrar o estado de pesquisa e as tendências de um determinado tópico (Fu et al., 2010).

Na próxima seção, são apresentados os métodos bibliométricos, coleção de dados e a análise de redes sociais (SNA). Na terceira seção, os resultados obtidos são sobre o desempenho geral e a dinâmica das publicações sobre o tema, países, instituições, autores, coautores e palavras-chave.

Sendo assim, este artigo tem como objetivo geral analisar a produção científica na área de climatologia urbana de forma sistêmica.

Os objetivos específicos são:

1. Identificar artigos e artigos de revisão publicados utilizando o termo "Urban Heat Island" OR "UHI", inserido em título, resumo e/ ou palavras-chave.

2. Identificar a quantidade de artigos e artigos de revisão utilizando o termo "Urban Heat Island* OR "UHI" AND "Local Climate Zones" OR "LCZ", inserido em título, resumo e/ ou palavras-chave.

3. Identificar a concentração dessa produção científica por país e por autor.

4. Elaborar uma sistematização dos principais critérios adotados nos artigos filtrados para o portfólio bibliográfico.

A partir desta pesquisa foi possível interpretar o grau de relevância deste termo para pesquisas em climatologia urbana e obter material de apoio para futuras pesquisas. 


\section{MATERIAL E MÉTODOS}

Para o alcance dos objetivos específicos deste trabalho foram realizadas as etapas de: seleção de critérios de busca; levantamento de dados; organização dos dados; a produção e a análise de gráficos e tabelas com os valores encontrados para cada critério selecionado. As etapas respeitaram a metodologia ProKnow-C (ENSSLIN et al., 2010).

De acordo com Santos, Schenatto e Oliveira (2017, p.3), o método de seleção do referencial bibliográfico denominado ProKnow-C (ENSSLIN et al., 2010) consiste em uma quantidade sequencial de procedimentos, sendo eles: "definição do mecanismo de busca de artigos científicos a ser utilizado, seguindo por uma série de procedimentos até atingir a fase de filtragem e seleção do portfólio bibliográfico relevante acerca do tema".

O primeiro termo utilizado para a busca de dados foi "Urban Heat Island*" OR "UHI", que em português é traduzido como Ilha de Calor Urbana (ICU), sendo o termo mais abrangente para a pesquisa por ser a denominação atual para anomalias e distribuição térmica no perímetro urbano e o que se pretende pesquisar mais detalhadamente nos próximos capítulos. Este termo foi analisado na base de dados Scopus.

Foram utilizados outros dois termos para nortear a filtragem durante a busca de dados, sendo eles: "Remote Sensing" e "Satellite Imagery". Estes retratam o cenário atual de pesquisas que trabalham com mapas temáticos, como as de climatologia urbana. O uso de sensoriamento remoto e com isso, imagens de satélite, vem sendo aplicado para planejamento urbano e ambiental das cidades, mostrando-se uma ferramenta importante para fiscalização, monitoramento, criação de cenários de qualificação e gestão.

Em seguida, analisaram-se os termos "Regression Analysis" e "GIS". De acordo com Foissard (2015), os modelos de regressão linear são largamente utilizados para espacialização de ilhas de calor urbanas. Como estes cálculos estatísticos são aplicados em softwares de geoprocessamento, optou-se pela busca também do termo "GIS".

Já o termo utilizado para a busca de dados mais afunilada foi "Local Climate Zones" OR "LCZ" por ser a denominação do método de classificação da paisagem para estudos de ilha de calor urbanas (e do campo térmico em geral da cidade), criado por STEWART (2011a). É um método que possui várias camadas de informação sobrepostas com intuito de ordenar a diversidade de temperatura através da identificação de classes. O método LCZ foi criado com o intuito de auxiliar na comunicação e padronização de informações sobre ilhas de calor urbanas em nível global, sendo amplamente testado por pesquisadores de diversos países da Europa, dos Estados Unidos, da Austrália e do Brasil.

Os resultados foram obtidos a partir de filtragem, para o caso dos termos aparecerem em: título, resumo, palavra-chave. As duplicidades foram eliminadas e houve a verificação da aderência do termo ao título, ao resumo e ao texto, e a verificação da disponibilidade da publicação.

O levantamento de dados do termo "Local Climate Zones" OR "LCZ" foi feito por meio das bases: Scopus, Web of Science, Science Direct, Wiley Online Library, Scielo e IEEE Xplore; a sistematização dos dados foi realizada em 
planilhas do software EndNote, versão X8 (teste), para posterior processamento dos dados (Figura 2).

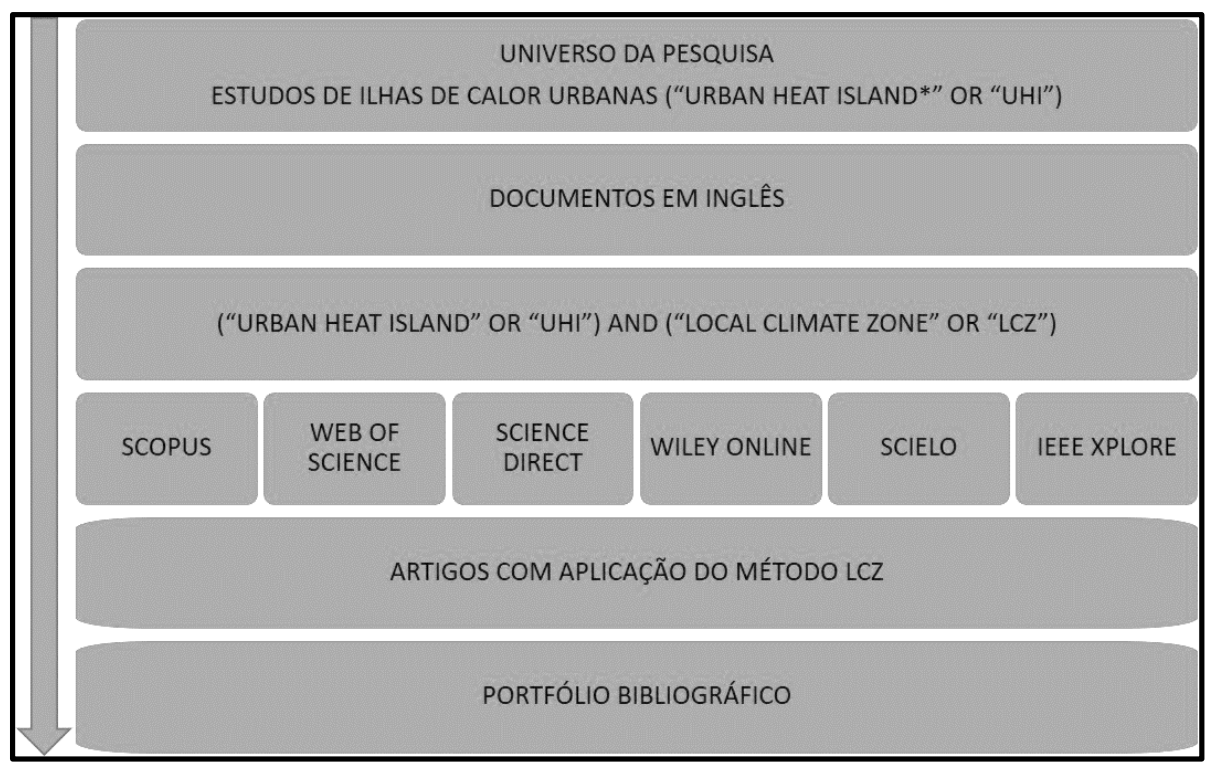

Figura 2 - Fluxograma de sistematização dos dados da pesquisa.

A análise bibliométrica investiga publicações científicas por meio de uma série de procedimentos, como publicações de diferentes periódicos, países, instituições, autores e análise de citações, além de identificar caminhos mais centrados na análise de conteúdo e evolução da pesquisa (Liu et al., 2011).

O software VOSviewer foi utilizado para sistematização de dados. Permite construir e visualizar redes bibliométricas, incluindo por exemplo, periódicos e pesquisadores, baseando-se nas relações de cocitação ou coautoria. Há também a funcionalidade de mineração de texto, por meio da qual se constrói e visualiza redes de coocorrência entre termos extraídos da literatura científica, facilitando a análise detalhada de características de resultados de pesquisas relacionadas.

O método SNA é uma maneira visual de analisar conexões entre autores (pessoas ou grupos) o que reflete, estatisticamente, a centralidade dos autores e a força da intensidade das relações entre eles (Newman, 2001).

Baseando-se na teoria de redes e softwares específicos, o SNA tem sido amplamente utilizado para medir a colaboração de pesquisa em vários campos do conhecimento, de modo que as contribuições de diferentes países, instituições e cientistas possam ser avaliadas (Nunkoo et al., 2013). Constituem softwares de visualização frequentemente usados para o SNA o: Pajek, Gephi, UCINET, Citespace II, VOSviewer e SciMAT.

Neste estudo, o SNA foi usado para avaliar a colaboração acadêmica entre diferentes países e autores através da aplicação do VOSviewer. Softwares que usam a técnica de mapeamento VOS (Van Eck et al., 2007), onde "VOS" significa visualização de similaridades (visualization of similarities). Os mapas são bidimensionais e baseados em distância, ou seja, a distância entre dois itens reflete a força da relação entre eles. Uma distância menor geralmente indica uma relação mais forte. 


\section{RESULTADOS E DISCUSSÃO}

A partir da base de dados Scopus foi possível encontrar 3.247 artigos e artigos de revisão utilizando o termo ("urban heat island* OR "UHI"), sendo este valor limitado a documentos em inglês.

Filtrando por área temática ocorre sobreposição de documentos, mesmo assim, as que mais aparecem são Ciências da Terra e Planetárias (44,3\%), Ciência Ambiental (42,3\%). Em seguida encontram-se as áreas temáticas de Engenharia $(22,3 \%)$ e Ciências Sociais (21,9\%), respectivamente. Um percentual menor, mas ainda significativo, é atribuído para as áreas temáticas de Agricultura (11,3\%), Energia (10,6\%) e Ciências de Materiais (4\%).

Um percentual menor apareceu em áreas temáticas de Medicina (3,2\%), Física e Astronomia (2,9\%), Ciências da Computação $(2,1 \%)$ e Outros (10\%).

A data de pesquisa sem filtros obteve o primeiro artigo publicado no ano de 1967 (DAVIDSON, B., 1967) e o mais recente consta na base como data do próximo ano, 2019 (SEN, S., ROESLER, J., KING, D., 2019). Ainda pela busca, foi possível detectar que apenas 3,4\% deste total são artigos de revisão. A série histórica utilizada na pesquisa foi de 1967 até 2019.

Deste total apenas 150 são disponíveis sem custo, ou seja, 4,62\%. O que evidencia uma fragilidade de pesquisa na área de climatologia urbana e mais precisamente, sobre ilhas de calor urbanas (ICU). Esta dificuldade de acesso faz com que a divulgação de novas metodologias de coleta, processamento de dados e de classificação de distribuição térmica em áreas urbanas seja retardada ou, até mesmo, interrompida. Desta forma, há a necessidade vigente de publicar pesquisas com livre acesso para melhorar a padronização de informações a respeito deste assunto e com isso permitirá aprofundar cada vez mais as caracterizações de regiões urbanas no que tange as Ilhas de Calor Urbanas.

Ao analisar a quantidade de publicações nos primeiros 30 anos, são obtidos 256 registros de publicações na base Scopus, representando $7,88 \%$ do total e nenhum artigo de revisão sobre o assunto foi publicado neste período. Ainda neste período, 86 artigos publicados constam como país de origem indefinido. O país líder de publicações era os Estados Unidos com 68, China em quinto lugar com 7 artigos e o Brasil não aparecia no ranking. Observou-se também que OKE, T. R. liderava o número de publicações, com 13 artigos. Em seguida constava BALLING, R. C. com 6 artigos, e BRAZEL, S. W. e NKEMDIRIM, L.C. com 5 publicações cada. CHING, J. K. S. constava em quinto lugar com 4 publicações, mesmo número dos demais autores até a décima colocação conforme Quadro 2. 
Quadro 2 - Número de publicações por autor no período de 1967 - 1996.

\begin{tabular}{|l|l|}
\hline Oke, T.R. & 13 \\
\hline Balling, R.C. & 6 \\
\hline Brazel, S.W. & 5 \\
\hline Nkemdirim, L.C. & 5 \\
\hline Ching, J.K.S. & 4 \\
\hline Clarke, J.F. & 4 \\
\hline Gallo, K.P. & 4 \\
\hline Goldreich, Y. & 4 \\
\hline Karl, T.R. & 4 \\
\hline Tarpley, J.D. & 4 \\
\hline
\end{tabular}

Ao analisar o resultado da busca por ano de publicação, percebe-se que de 1997 até o presente constam 2.991 publicações, o que representa $92,12 \%$ do total. Por este motivo no gráfico da Figura 3 aparece a barra em cor laranjada com um apanhado de todas as publicações anteriores a 1997.

Há um aumento acelerado nas publicações a partir do ano de 2008, conforme gráfico da figura 3. No quadriênio de 2008 a 2012 o número de publicações duplicou, assim como no quadriênio posterior, de 2012 a 2016 . O ano de 2017 ainda consta como o mais produtivo, tendo em vista que a análise de 2018 não está completa, comprovando o aumento no número de publicações no período.

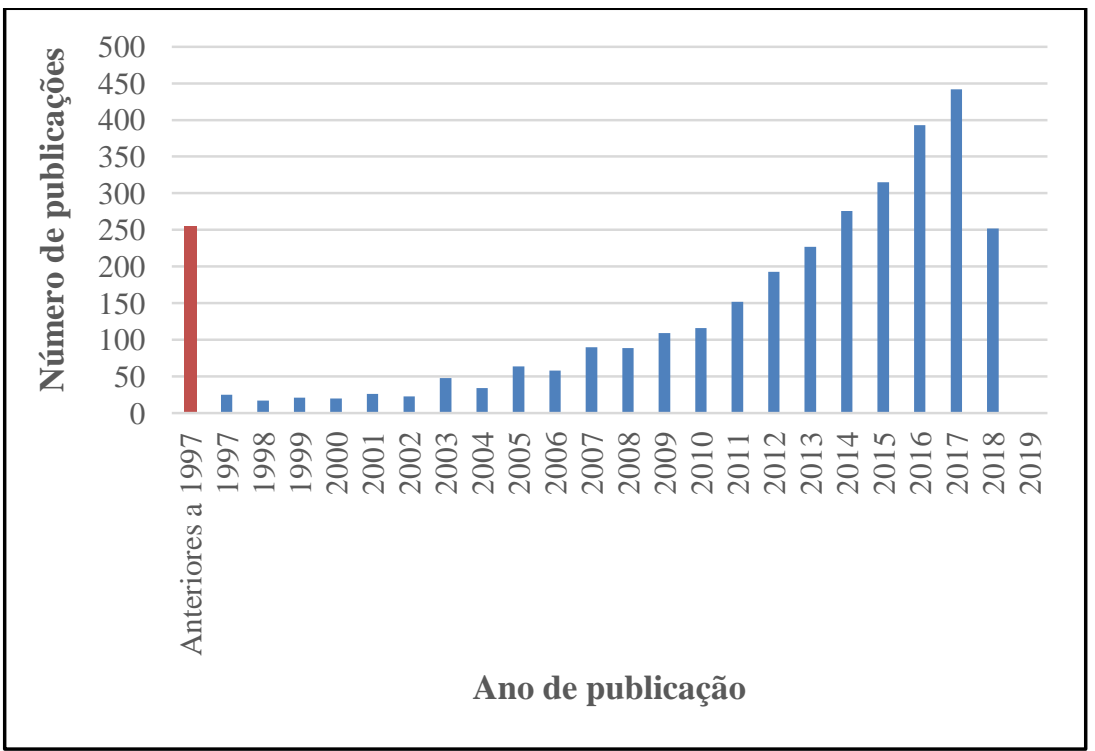

Figura 3 - Número de publicações por ano a partir da busca "urban heat island*" OR "UHI" na base de dados Scopus. *última busca no Scopus em 18 de junho de 2018.

De modo global, os autores que estão em evidência em climatologia urbana e mais especificamente em estudos de Ilhas de Calor estão 
representados na Figura 4 elaborada a partir do software VOSViewer com a base de dados Scopus.

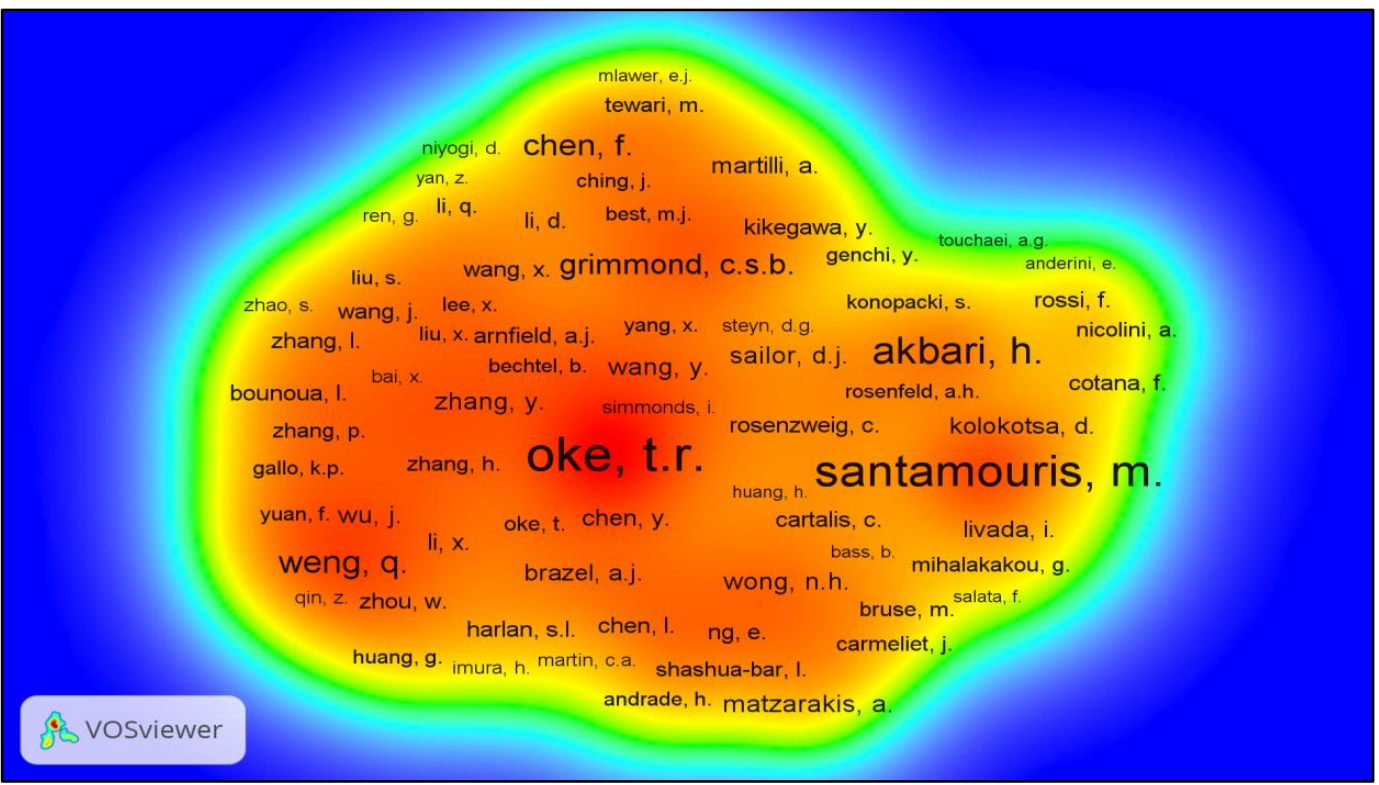

Figura 4 - Termos encontrados a partir de filtragem no software VOSViewer a partir da base de dados Scopus.

Ao analisar as ligações entre autores (Figura 5), é possível perceber os grupos existentes e suas conexões. O principal grupo de estudos tem à frente Oke, T. R. conectado aos demais grupos de estudos. Santamouris, M. e Akbari, $\mathrm{H}$. estão à frente da segunda maior rede de pesquisas nesta área.

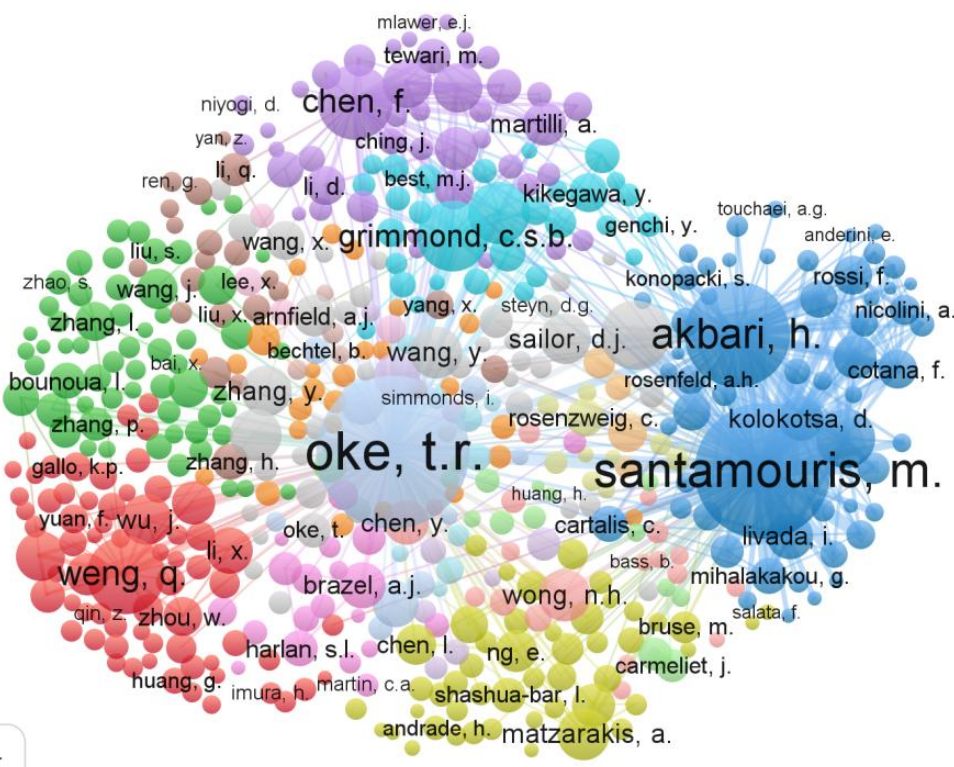

Figura 5 - Grupos de autores e suas conexões. Elaborado a partir de filtragem no software VOSViewer a partir da base de dados Scopus. 
Dentre os países que mais publicaram neste período, Estados Unidos continuam em primeiro lugar (com 788 artigos) e China em segundo lugar (com 573 artigos). Observou-se também que o autor que mais publicou neste período foi SANTAMOURIS, M., conforme Quadro 3.

Quadro 3 - Número de publicações por autor no período de 1997 - 2019.

\begin{tabular}{|l|l|}
\hline Santamouris, M. & 46 \\
\hline Pisello, A.L. & 29 \\
\hline Weng, Q. & 25 \\
\hline Brazel, A.J. & 25 \\
\hline Cotana, F. & 24 \\
\hline Masson, V. & 21 \\
\hline Wong, N.H. & 20 \\
\hline Akbari, H. & 18 \\
\hline Baik, J.J. & 18 \\
\hline Davies, M. & 18 \\
\hline
\end{tabular}

Além das palavras-chave esperadas (ilha de calor e ilha de calor urbana, temperatura atmosférica, área urbana, urbanização, clima urbano), foram encontradas também com alta frequência: planejamento urbano, sensoriamento remoto e uso do solo, o que mostra um cenário de revolução digital no que tange o planejamento e uso do solo em cidades, amplamente discutido na literatura atual (Figura 6).

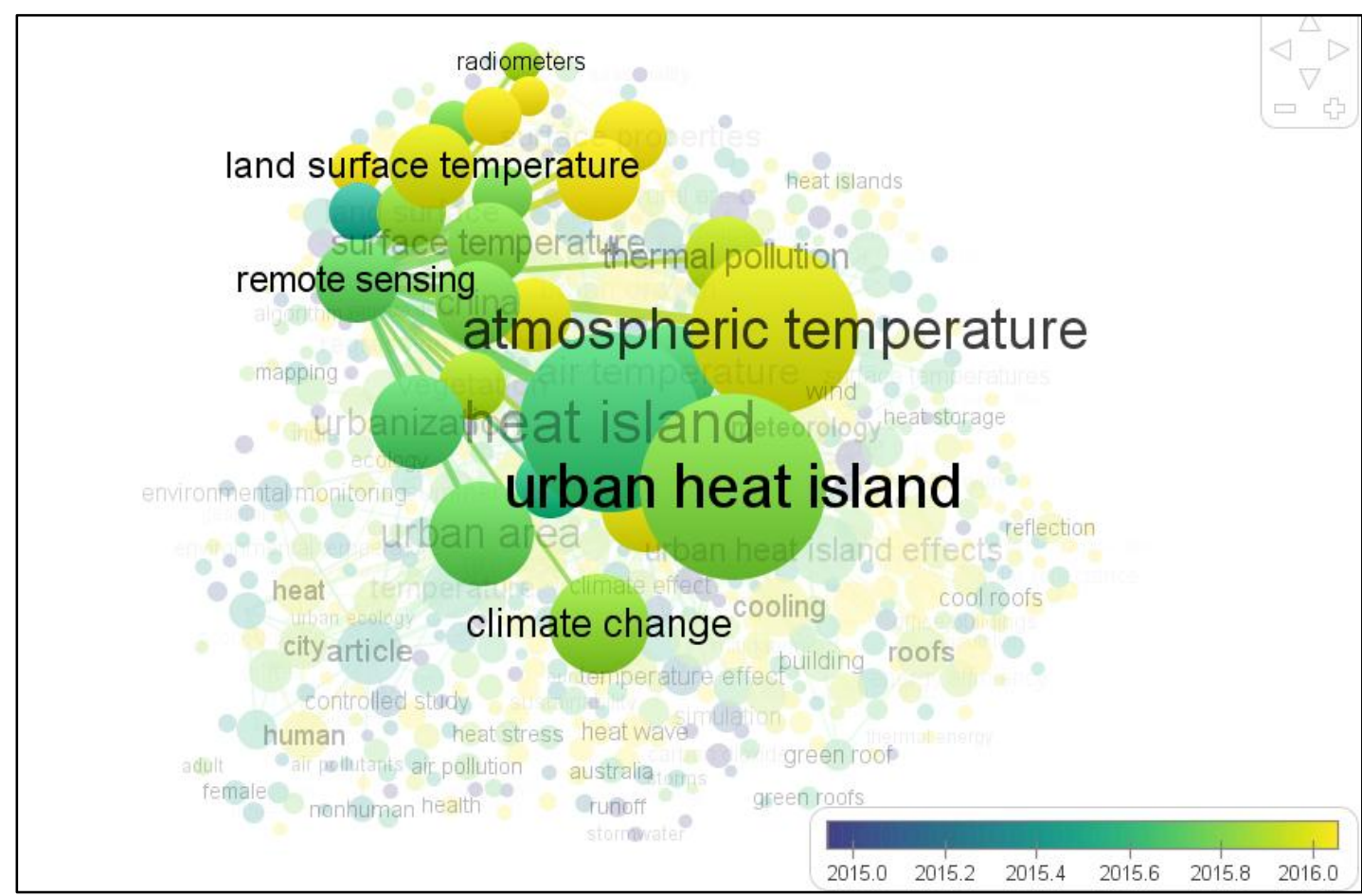

Figura 6 - Termos encontrados a partir de filtragem no software VOSViewer a partir da base de dados Scopus. 
O termo "Remote Sensing" (sensoriamento remoto) aparece a partir do ano de 1989, o que demonstra a aplicação de técnicas de sensoriamento remoto em estudos de ICUs há praticamente 30 anos. Já o termo "Satellite Imagery" (imagens de satélite), tem início nesta filtragem de buscas a partir do ano de 1999. Percebe-se então, um forte crescimento do uso de geotecnologias para a identificação, classificação e mensuração das ilhas de calor urbanas.

Em seguida, analisou-se os termos "Regression Analysis" e "GIS". Estes foram encontrados entre as palavras-chave na base de dados Scopus e demonstram que o método de cálculo matemático e o processamento de imagens de satélite são abordados atualmente quando se trata de estudos de clima urbano. Este tipo de informação corrobora a escolha de métodos contemporâneos para o processamento de dados sobre ICU, tendo a finalidade de auxiliar na elaboração de uma pesquisa inovadora.

E por fim, o termo ("Urban Heat Island*" OR "UHI") AND ("Local Climate Zone*" OR "LCZ") foi analisado em seis (6) bases de dados: Scopus, Web of Science, Science Direct, Wiley Online Library, Scielo e IEEE Xplore. Obteve-se um total de 67 artigos e artigos de revisão disponíveis. Destes, 57 foram utilizados para o portfólio bibliográfico (PB) por terem como foco a aplicação do método LCZ. Sendo feita a sistematização dos dados em formato de tabela.

Com o portfólio bibliográfico em mãos foi possível perceber que 24 deles possuem 10 citações ou mais (Figura 7). Feita uma análise da relevância científica simplificada, identificam-se alguns destaques: o artigo de Stewart, Oke e Krayenhoff (2014) possui 126 citações e o artigo de Bechtel et al. (2015) possui 113 citações no Google Acadêmico, sendo os únicos no portfólio com mais de 100 citações. 


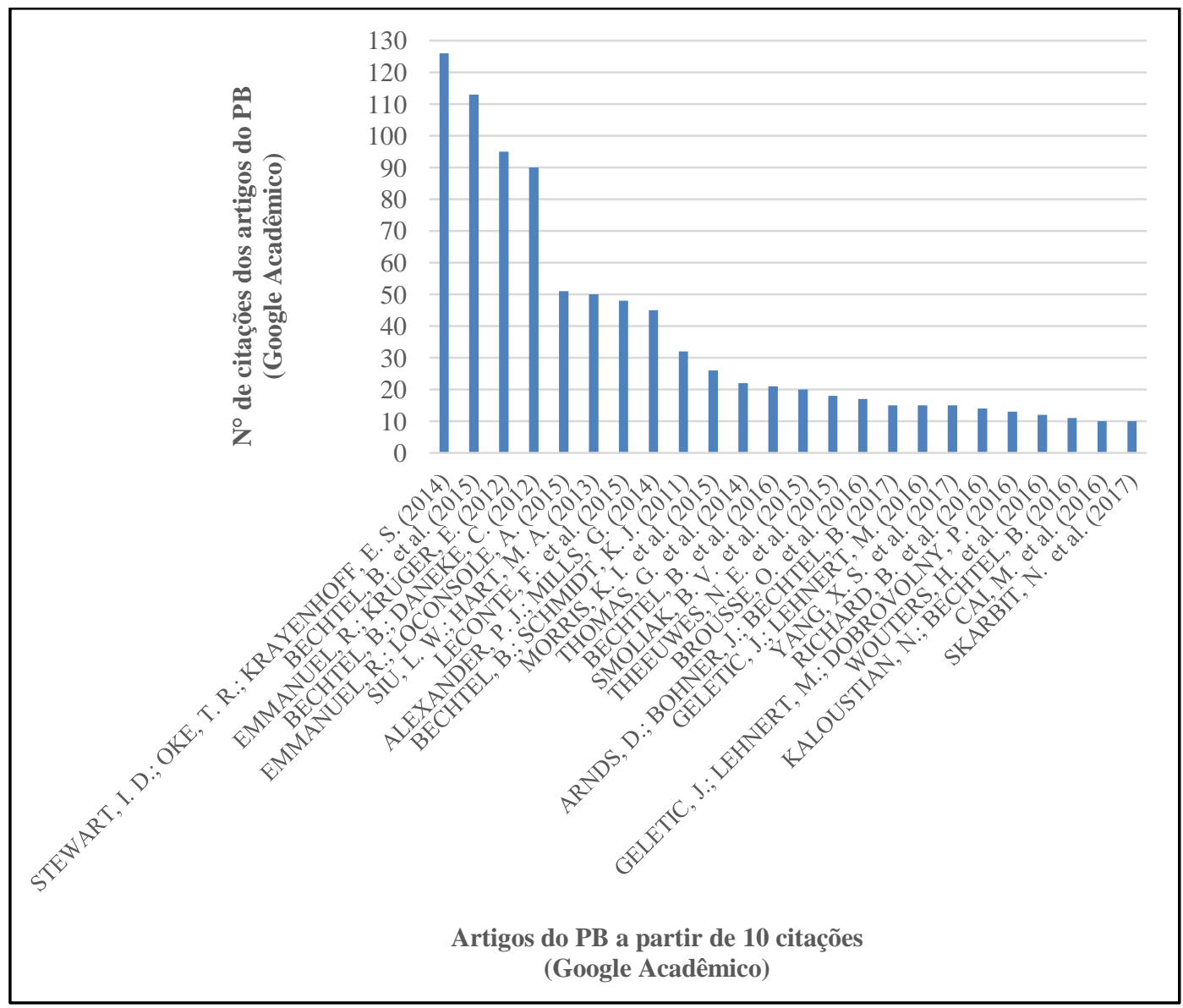

Figura 7 - Relevância dos artigos do PB no Google Acadêmico.

A partir dos dez artigos mais citados, foram analisados os critérios iniciais para a sistematização dos dados, como: cidades e países de aplicação do método LCZ, as características urbanas encontradas, a magnitude da ilha de calor urbana encontrada em cada artigo, os métodos de coleta de dados higrotérmicos, simulação computacional e métodos de cálculo estatístico aplicados nas pesquisas e, por fim, o software utilizado para processamento de dados.

A partir da leitura dos textos na íntegra foi possível detectar quais parâmetros mais aparecem nos artigos e quais as informações mais importantes para o estudo de ilhas de calor urbanas, resultando nas colunas da tabela de sistematização dos dados (Tabela 4). Cada linha da tabela representa um dos 58 artigos analisados. 
Tabela 4 - Proposta de sistematização para os dados dos artigos filtrados.

\begin{tabular}{|c|c|c|c|c|c|c|c|c|c|c|c|c|}
\hline 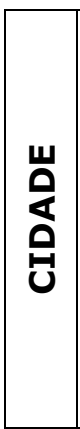 & $\sum_{\vec{J}}^{\overleftarrow{J}}$ & 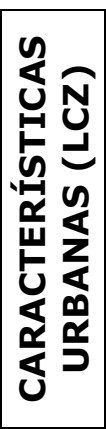 & 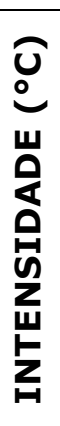 & 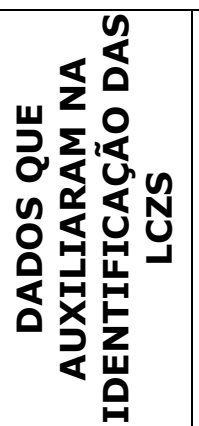 & 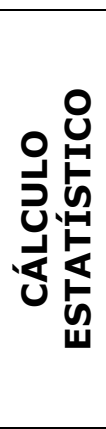 & 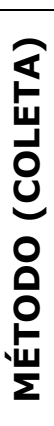 & 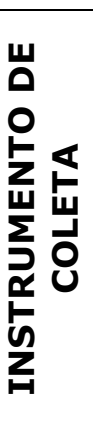 & 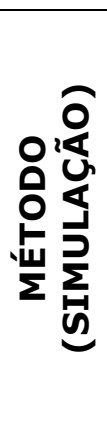 & 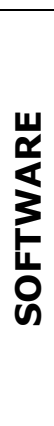 & 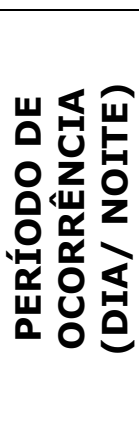 & 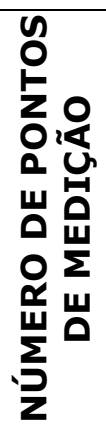 & 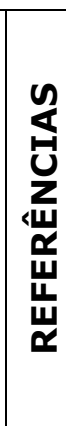 \\
\hline
\end{tabular}

Desta forma, foi possível detectar as cidades e países de maior ocorrência de aplicação do método, bem como seus respectivos climas (Figura 8). Servindo como embasamento para os resultados de coletas e análises de caráter inédito da aplicação do método LCZ em cidades brasileiras.

Para esta etapa foram lidos 28 artigos, sendo estes os que possuem maior relevância. Europa e Ásia são os continentes de maior aplicação do método de classificação da paisagem LCZ, seguidas respectivamente pela América do Norte e África. Em relação aos climas nos quais ocorre aplicação do método, foi possível detectar que os artigos de maior relevância não utilizaram como área de estudos a América do Sul e mais especificamente, o Brasil. De acordo com a classificação de Köppen-Geiger, os climas de maior aplicação são Cfb (9 artigos), Cfa (5 artigos) e Dfa (4 artigos), respectivamente.

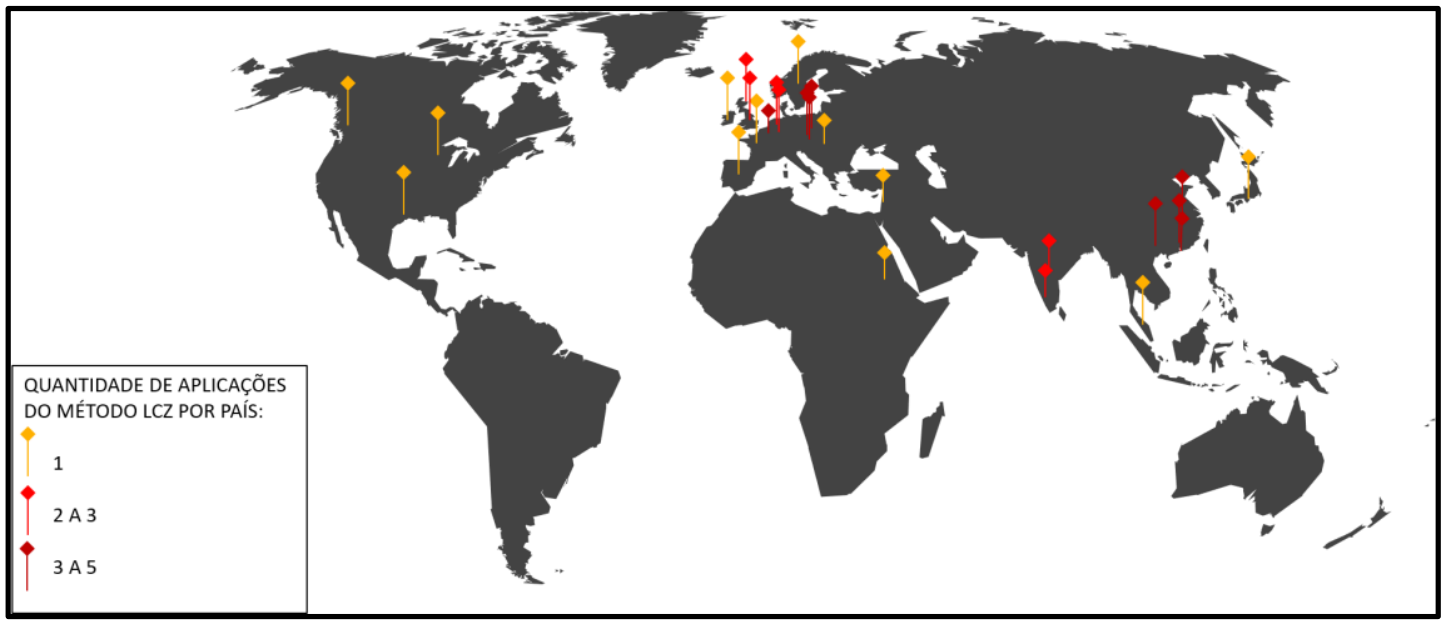

Figura 8 - Locais de aplicação do método LCZ.

Também foi possível detectar as características urbanas encontradas, ou seja, as zonas climáticas locais (LCZ) foram identificadas até o momento, nas cidades analisadas em cada um dos artigos, colaborando para a interpretação do sucesso de adaptação do método aos diferentes países onde já foi aplicado, incluindo países da Europa, Ásia e África. A partir do gráfico da Figura 9 é possível perceber que as classes que mais aparecem nos artigos são LCZ 2, LCZ 6 e LCZ 5 que representam respectivamente áreas de edificações compactas de média elevação, abertas de média elevação e abertas de baixa elevação. 


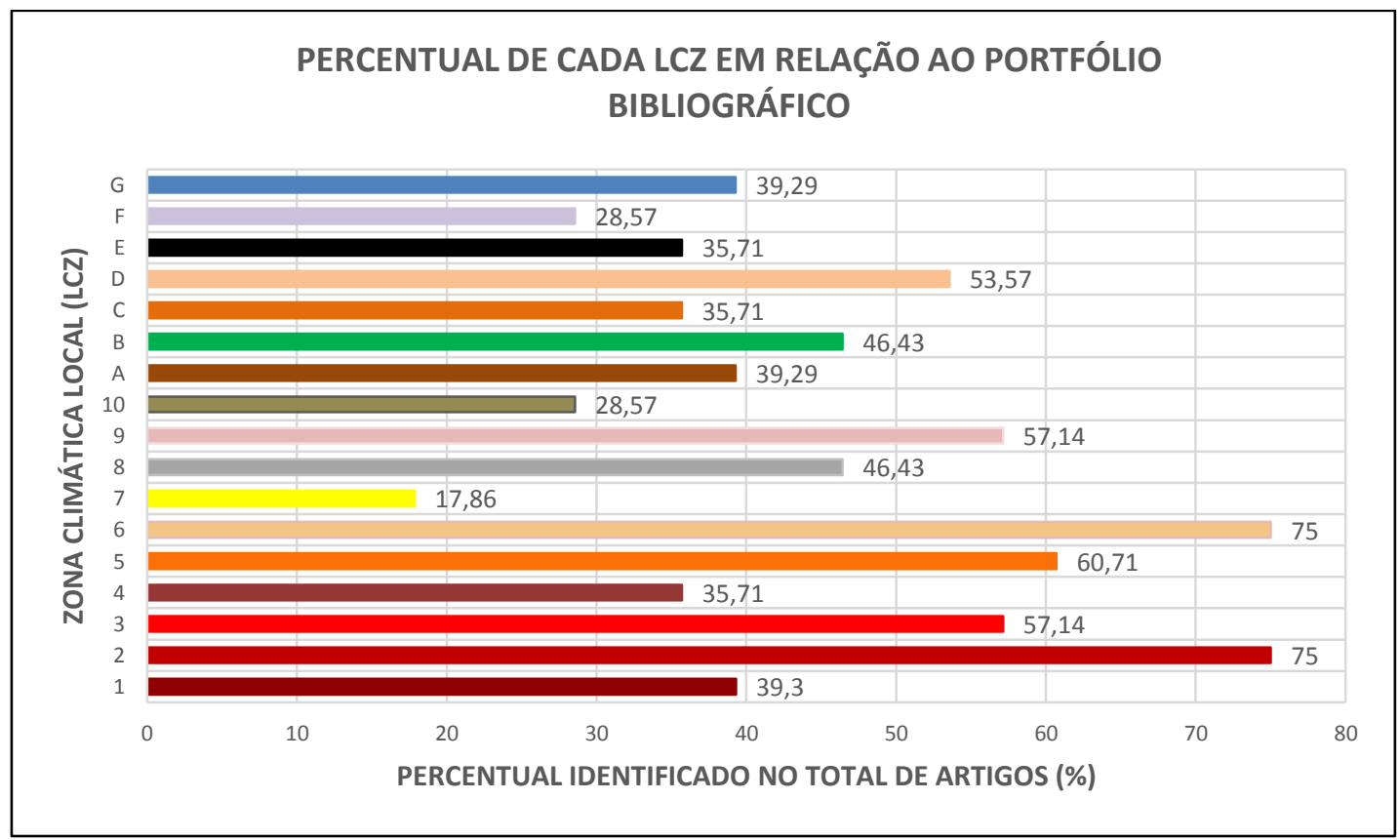

Figura 9 - Percentual de cada LCZ em relação ao Portfólio Bibliográfico.

Conforme o porfólio bibliográfico podemos verificar que Nagano, Vancouver, Upsala, Glasgow, Hamburgo, Dublin, Bélgica, Nancy, Hong Kong, Kochi, Beirute, Szeged, Putrajaya, Khartoum, Birmingham, Minneapolis, Saint Paul, Praga, Brno e Nagpur são cidades que não possuem toda a diversidade de classes (17 zonas climáticas locais) que o método propõe como passíveis de serem identificadas. Nelas ocorre grande repetição da Classe 3, que corresponde a áreas muito compactadas e com edificações de baixa elevação. Cabe salientar que $67,90 \%$ dos artigos analisados utilizam menos de 10 classes dentre as 17 zonas climáticas locais propostas por STEWART (2012).

Outro parâmetro analisado foi a magnitude da ilha de calor urbana (ICU) encontrada conforme tabela 5.

Tabela 5 - Dados de ICU por cidade dos artigos do Portfólio Bibliográfico. Amarelo: ICU de baixa magnitude. Laranja: ICU de média magnitude. Vermelho: ICU de alta magnitude. Classificação climática de acordo com Köppen-Geiger, por cidade dos artigos do Portfólio Bibliográfico.

\begin{tabular}{|l|c|c|}
\hline CIDADE/ PAÍs & ICU ( $\left.{ }^{\circ} \mathrm{C}\right)$ & $\begin{array}{c}\text { CLIMA } \\
\text { KÖPPEN }\end{array}$ \\
\hline Hamburgo, Alemanha, Dublin, Irlanda, e Houston, EUA. & não cita & $\begin{array}{c}\text { Dfa; Csb; } \\
\text { Dfb }\end{array}$ \\
\hline Hamburgo, Alemanha & não cita & $\begin{array}{c}\text { Cfb; Dfa; } \\
\text { Cfa }\end{array}$ \\
\hline Glasgow, Escócia & não cita & Am \\
\hline Nancy, nordeste da França & não cita & Cfb \\
\hline Khartoum, Sudão & não cita & Am \\
\hline Brno, Hradec Králové, Olomouc, República Tcheca & não cita & Cfa \\
\hline Praga e Brno, República Tcheca & não cita & Cfb \\
\hline
\end{tabular}




\begin{tabular}{|l|c|c|} 
Guangzhou, China & não cita & Dfa \\
\hline Hong Kong, China & não cita & Cfb \\
\hline Madrid, Espanha & não cita & Af \\
\hline Hamburgo, Alemanha & 1 & Am \\
\hline Birmingham, Inglaterra & 1,2 & Csa \\
\hline Hamburgo, Alemanha & 2 & Dfa \\
\hline Glasgow e entorno, Escócia. & 2 & Csa \\
\hline Madrid e seu entorno, Espanha & 2 & Csa \\
\hline Nanjing, China & 3 & Cfb \\
\hline Putrajaya, Malásia & 3,1 & Cfb;Dfb; \\
\hline Hong Kong SAR, China & 3,8 & Cfa \\
\hline Minneapolis, St. Paul, e entorno & 4 & Cfb \\
\hline Szeged, Hungria & 4 & Cfb \\
\hline Dublin, Irlanda & 4 & Cfb \\
\hline Todas as cidades, Bélgica & 4 & Csa \\
\hline Nagpur, India & 4,1 & Cfa \\
\hline Kochi, India & 4,6 & Cfb \\
\hline Nagano, Japão; Vancouver, Canadá; Upsala, Suécia & 5 & Aw \\
\hline Beirute, Líbano & 6 & Cfa \\
\hline
\end{tabular}

Isso é relevante pois o método define diferenças térmicas intraurbanas, ou seja, nos diferentes ambientes urbanos existentes; e quanto mais acentuada a diferença de temperatura entre um ponto e outro da cidade, significa que mais válido é o método, já que ele proporciona um nível de detalhamento muito maior que os métodos tradicionais de mensuração de ICU (diferença térmica entre zona urbana e zona rural).

Cabe ainda salientar que os climas identificados na tabela 5 são encontrados no Brasil, servindo de base para futuras comparações in loco ou por sensoriamento remoto.

No que tange a simulação computacional e métodos de cálculo estatístico aplicados nas pesquisas, foi possível perceber que estão diretamente ligados ao número de pontos coletados e a análise do raio do entorno de cada ponto coletado. Todos os artigos analisados nesta fase final descreveram a simulação computacional utilizada para gerar seus mapas e realizar suas análises estatísticas e $100 \%$ deles utilizaram métodos estatísticos para tratamento dos pixels.

Além disso, foi analisado o software utilizado, dando um indicativo do percentual de publicações disponíveis realizadas e identificadas com programas computacionais gratuitos e as cidades onde foram feitas as aplicações do método conforme Tabela 6 . Foi possível verificar que $32,14 \%$ ( 9 artigos) utilizaram software gratuito para a pesquisa, 14,29\% (4 artigos) utilizaram software pago e a maioria dos autores filtrados neste trabalho 46,43\% (13 
artigos) não citam o software utilizado para gerar os mapas das Zonas Climáticas Locais (LCZ).

Tabela 6 - Artigos do Portfólio Bibliográfico em ordem de número de citações; lista de tipos de software utilizados em cada artigo.

\begin{tabular}{|l|c|c|}
\hline CIDADE/ PAÍS DE APLICAÇÃO DO MÉTODO & SOFTWARE & $\begin{array}{l}\text { No CITAÇÕES } \\
\text { DOS ARTIGOS }\end{array}$ \\
\hline $\begin{array}{l}\text { Nagano, Japão; Vancouver, Canadá; Upsala, } \\
\text { Suécia }\end{array}$ & não cita & 126 \\
\hline $\begin{array}{l}\text { Hamburgo, Alemanha, Dublin, Irlanda, e } \\
\text { Houston, EUA. }\end{array}$ & Gratuito & 113 \\
\hline Glasgow e entorno, Escócia. & não cita & 95 \\
\hline Hamburgo, Alemanha & Gratuito & 90 \\
\hline Glasgow, Escócia & Pago & 51 \\
\hline Hong Kong SAR, China & não cita & 50 \\
\hline Nancy, nordeste da França & Gratuito & 48 \\
\hline Dublin, Irlanda & Gratuito & 45 \\
\hline Hamburgo, Alemanha & Gratuito & 32 \\
\hline Putrajaya, Malásia & Gratuito & 26 \\
\hline Kochi, India & não cita & 22 \\
\hline Khartoum, Sudão & Gratuito & 21 \\
\hline Minneapolis, St. Paul, e entorno & não cita & 20 \\
\hline Madrid e seu entorno, Espanha & não cita & 18 \\
\hline Madrid, Espanha & não cita & 17 \\
\hline Hamburgo, Alemanha & não cita & 15 \\
\hline Brno, Hradec Králové, Olomouc, República & não cita & 15 \\
\hline Tcheca & Pago & 15 \\
\hline Nanjing, China & não cita & 14 \\
\hline Birmingham, Inglaterra & não cita & 13 \\
\hline Praga e Brno, República Tcheca & Pago & 12 \\
\hline Beirute, Líbano & Gratuito & 11 \\
\hline Guangzhou, China & Gratuito & 10 \\
\hline Szeged, Hungria & não cita & 10 \\
\hline Nagpur, Índia & Pago & 9 \\
\hline Hong Kong, China & não cita & 9 \\
\hline
\end{tabular}

Ficou demonstrado que a maior concentração de uso de softwares gratuitos ocorre na Europa. É importante salientar que a citação do programa computacional na metodologia faz com que o estudo seja reproduzido com maior facilidade pelos pesquisadores ao redor do mundo. O percentual referente ao uso de programas gratuitos é relevante e faz com que as pesquisas de climatologia urbana sejam acessíveis, gera também uma probabilidade de padronização das pesquisas nesta área. 


\section{CONCLUSÃO}

A partir da quantidade de publicações encontradas na base Scopus sobre ilha de calor urbano (3247) e o amplo crescimento de publicações ao longo dos últimos vinte (20) anos, que representa mais de $90 \%$ do total de publicações, é possível perceber a importância do tema.

Uma grande preocupação dos pesquisadores dessa área temática é o fato de que não há uma padronização e o nível de detalhamento da distribuição térmica em escala urbana é muitas vezes desconsiderado. No que tange o método de classificação da paisagem aqui analisado, a grande ocorrência de citações dos artigos publicados desde seu surgimento (STEWART, 2011-a) mostra a efetividade de sua aplicação tendo em vista que nos estudos considerados modernos, a partir dos anos 1950, representa um esforço de aprimoramento no nível de detalhamento e padronização de coleta, descrição e critérios de mensuração. A sistematização de dados realizada é importante para ilustrar de forma didática como o presente método LCZ ainda é passível de diversos testes e aprimoramentos.

Deste modo, este artigo colabora com as pesquisas em climatologia urbana e cria um conjunto de dados organizados que auxiliam no planejamento de futuras pesquisas nesta área.

\section{REFERÊNCIAS BIBLIOGRÁFICAS}

AMORIM, M. C. C. T. O.; MONTEIRO, A. As temperaturas intraurbanas: exemplos de Brasil e Portugal. Confins, Revista Franco-Brasileira de Geografia, São Paulo, v.10, n.13, p.1-17, 2011.

AMORIM, M. C. C. T.; DUBREUIL, V.; CARDOSO, R. S. Modelagem espacial da ilha de calor urbana em Presidente Prudente (SP) - Brasil. Revista Brasileira de Climatologia, v. 16, p. 29-45, 2015.

ARNFIELD, A. J. Two decades of urban climate research: A review of turbulence, exchanges of energy and water and the urban heat island. International Journal of Climatology, 23: p. 1-26, 2003. DOI: 10.1002/joc.859.

ENSSLIN, L. et al. ProKnow-C, Knowledge Development Process Constructivist: processo técnico com patente de registro pendente junto ao INPI. Brasil: [s.n.], 2010a.

FOISSARD, X. L'îlot de chaleur urbain et le changement climatique: application à I'agglomération rennaise. 2015. 248f. Tese (Doutorado em Geografia) Université Rennes 2, Rennes, França.

Fu, H.Z.; Ho, Y.S.; Sui, Y.M. \& Li, Z.S. 2010. A bibliometric analysis of solid waste research during the period 1993-2008. Waste Management, 30: 24102417.

Hirsch, J.E. 2005. An index to quantify an individual's scientific research output. P. Natl. Proceeding of the National Academy of Sciences of the United States of America, 102: 16569-16572.

Liu, X.; Zhang, L. \& Hong, S. 2011. Global biodiversity research during 19002009: a bibliometric analysis. Biodiversity and Conservation, 20: 807-826. 
Newman, M.E. 2001. Scientific collaboration networks. I. Network construction and fundamental results. Physical Review Journals, 64(1): 016131.

MASSON, V. Adapting cities to climate change: A systemic modeling approach. Urban Climate, Paris, v. 10, p. 407-429, 2014.

MCCARTHY M. P.; BEST M. J.; BETTS R. A. Climate change in cities due to global warming and urban effects. Geophysical Research Letters, Washington, v. 37, p. $1-5,2010$.

MORRIS, C.J.G.; SIMMONDS, I. Associations between varying magnitudes of the urban heat island and the synoptic climatology in Melbourne, Australia. International Journal of Climatology, v. 20, n. 15, p. 1931-1954, 2000. Disponível em: <https://rmets.onlinelibrary.wiley.com/doi/abs/10.1002/10970088\%28200012 \%2920\%3A15\%3C1931\%3A\%3AAID-JOC578\%3E3.0.CO\%3B2-D>.

Nunkoo, R.; Gursoy, D. \& Ramkissoon, H. 2013. Developments in hospitality marketing and management: social network analysis and research themes. Journal of Hospital Marketing \& Management, 22: 269-288.

OKE, T. R. Boundary Layer Climates. London: Methuen \& Co. 2nd edn. 1987, 435p.

OKE, T. R. Towards better scientific communication in urban climate. Theorical and Applied Climatology. V. 84, p.179-190, 2006.

SANTOS, A. I.; SCHENATTO, F. J. A.; OLIVEIRA, G. A. Metodologia PROKNOW-C para construir o conhecimento acerca de previsão de demanda utilizando séries temporais. In: VII Congresso Brasileiro de Engenharia de Produção, 2017, Ponta Grossa. Anais do VII Congresso Brasileiro de Engenharia de Produção. Ponta Grossa: APREPRO, 2017.

SOUZA, C. A. Análise do conforto térmico de parques urbanos de recreação e de suas áreas de influência na cidade de Campo Grande - Mato Grosso do Sul. 2016. 140f. Dissertação (Mestrado em Meio Ambiente e Desenvolvimento Regional). Universidade Anhanguera-Uniderp, Campo Grande.

STEWART, I. D. Influence of meteorological conditions on the intensity and form of the urban heat island in Regina. The Canadian Geographer. v. 3, n. 44, p. 271-285, 2000.

STEWART, I. D. Redefining the urban heat island. 2011-a. 368f. Tese (Doutorado em Filosofia). The Faculty of Graduate Studies, The University of British Columbia, Vancouver.

STEWART, I. D.; OKE, T. R. Local Climate Zones for Urban Temperature Studies. Bull. American Meteorological Society, v. 93, p. 1879-1900, 2012. doi: 10.1175/BAMS-D-11-00019.1

Van Eck, N.J. \& Waltman, L. 2007. VOS: A new method for visualizing similarities between objects. In: DECKER. R, LENZ, H.J (eds). Advances in data analysis: Studies in Classification, Data Analysis and Knowledge Organization, Berlin, Heidelberg, Springer, p. 299-306.

VOOGT, J. A.; OKE, T. R. Thermal remote sensing of urban climates. Remote Sensing of Environment. v. 3, n. 86, p. 371-384, 2003. 
YE, Q., SONG, H. \& LI, T. Cross-institutional collaboration networks in tourism and hospitality research. Tourism Management Perspectives, 2(3): 55-64, 2012 